\title{
Weighted Discrete Universality of the Riemann Zeta-Function
}

\author{
Antanas Laurinčikas ${ }^{a}$, Darius Šiaučiūnas ${ }^{b}$ and \\ Gediminas Vadeikis ${ }^{a}$ \\ ${ }^{a}$ Institute of Mathematics, Faculty of Mathematics and Informatics, Vilnius \\ University \\ Naugarduko str. 24, LT-03225 Vilnius, Lithuania \\ ${ }^{b}$ Institute of Regional Development, Siauliai University \\ P. Višinskio str. 25, LT-76351 Šiauliai, Lithuania \\ E-mail(corresp.): darius.siauciunas@su.lt \\ E-mail: antanas.laurincikas@mif .vu.lt \\ E-mail: gediminas.vadeikis@mif.vu.lt
}

Received June 5, 2019; revised October 18, 2019; accepted October 18, 2019

\begin{abstract}
It is well known that the Riemann zeta-function is universal in the Voronin sense, i.e., its shifts $\zeta(s+i \tau), \tau \in \mathbb{R}$, approximate a wide class of analytic functions. The universality of $\zeta(s)$ is called discrete if $\tau$ take values from a certain discrete set. In the paper, we obtain a weighted discrete universality theorem for $\zeta(s)$ when $\tau$ takes values from the arithmetic progression $\{k h: k \in \mathbb{N}\}$ with arbitrary fixed $h>0$. For this, two types of $h$ are considered.
\end{abstract}

Keywords: approximation of analytic functions, Mergelyan theorem, Riemann zetafunction, universality, weak convergence.

AMS Subject Classification: 11M06; 41A30.

\section{Introduction}

The Riemann zeta-function $\zeta(s), s=\sigma+i t$,

$$
\zeta(s)=\sum_{m=1}^{\infty} \frac{1}{m^{s}}, \quad \sigma>1,
$$

since Riemann's and even Euler's times surprises mathematicians by the extensive field of applications and denseness of the set of its values. It is well

Copyright (c) 2020 The Author(s). Published by VGTU Press

This is an Open Access article distributed under the terms of the Creative Commons Attribution License (http://creativecommons.org/licenses/by/4.0/), which permits unrestricted use, distribution, and reproduction in any medium, provided the original author and source are credited. 
known the role of $\zeta(s)$ in the theory of distribution of prime numbers and in other problems of arithmetic, however, we, in this paper, prefer the denseness properties of $\zeta(s)$.

In the second decade of the last century, H. Bohr discovered [4] that the function $\zeta(s)$ takes every non-zero value infinitely many times in the strip $\{s \in \mathbb{C}: 1<\sigma<1+\delta\}$ with any $\delta>0$. H. Bohr and R. Courant proved [5] that, for fixed $\sigma, \frac{1}{2}<\sigma \leqslant 1$, the set

$$
\{\zeta(\sigma+i t): t \in \mathbb{R}\}
$$

is dense in $\mathbb{C}$. S.M. Voronin significantly generalized the above results. He obtained [25] that the set

$$
\left\{\left(\zeta\left(s_{1}+i \tau\right), \ldots, \zeta\left(s_{n}+i \tau\right)\right): \tau \in \mathbb{R}\right\}
$$

with any fixed numbers $s_{1}, \ldots, s_{n}, \frac{1}{2}<\operatorname{Re} s_{k}<1,1 \leqslant k \leqslant n$, and $s_{k} \neq s_{m}$ for $k \neq m$, and the set

$$
\left\{\left(\zeta(s+i \tau), \zeta^{\prime}(s+i \tau), \ldots, \zeta^{(n-1)}(s+i \tau)\right): \tau \in \mathbb{R}\right\}
$$

with every fixed $s, \frac{1}{2}<\sigma<1$, are dense in $\mathbb{C}^{n}$. However, a much more important merit of Voronin is his so-called universality theorem for the function $\zeta(s)$ [26]. This theorem asserts that a wide class of analytic functions can be approximated by shifts $\zeta(s+i \tau), \tau \in \mathbb{R}$. For a modern version of the Voronin universality theorem, it is convenient to use the following notation. Let $D=\left\{s \in \mathbb{C}: \frac{1}{2}<\sigma<1\right\}$. Denote by $\mathcal{K}$ the class of compact subsets of the strip $D$ with connected complements, and by $H_{0}(K), K \in \mathcal{K}$, the class of continuous non-vanishing functions on $K$ that are analytic in the interior of $K$. Then the following theorem is true.

Theorem 1. Let $K \in \mathcal{K}$ and $f(s) \in H_{0}(K)$. Then, for every $\varepsilon>0$,

$$
\liminf _{T \rightarrow \infty} \frac{1}{T} \operatorname{meas}\left\{\tau \in[0, T]: \sup _{s \in K}|\zeta(s+i \tau)-f(s)|<\varepsilon\right\}>0 .
$$

Here meas $A$ denotes the Lebesgue measure of a measurable set $A \subset \mathbb{R}$. By Theorem 1, the set of shifts $\zeta(s+i \tau)$ approximating a given function from $H_{0}(K)$ has a positive lower density, thus, it is infinite. Also, Theorem 1 can be considered as an infinite-dimensional version of the Bohr-Courant theorem on denseness of the set (1.1). The proof of Theorem 1 is given in [1] (in slightly different form), and in [9], [11], [24].

Theorem 1 is of continuous type: $\tau$ in $\zeta(s+i \tau)$ can take arbitrary real values. Also, a discrete version of Theorem 1 is known when $\tau$ takes values from a certain discrete set. Let $h>0$ be a fixed number.

Theorem 2. Let $K \in \mathcal{K}$ and $f(s) \in H_{0}(K)$. Then, for every $\varepsilon>0$,

$$
\liminf _{N \rightarrow \infty} \frac{1}{N} \#\left\{1 \leqslant k \leqslant N: \sup _{s \in K}|\zeta(s+i k h)-f(s)|<\varepsilon\right\}>0 .
$$


Here $\# A$ denotes the cardinality of the set $A$. The proof of Theorem 2 can be found in [22] and [1]. For shifts $\zeta\left(s+i k^{\alpha} h\right)$ with fixed $\alpha, 0<\alpha<1$, Theorem 2 is given in [6]. In $[15,21]$ and $[7,8,13,16]$, more general shifts of Dirichlet $L$-functions and Riemann zeta-function, respectively, were considered. We note that discrete universality theorems for zeta-functions sometimes are more convenient for practical applications, an example of this is the paper [3].

In [10], a weighted version of Theorem 1 was proposed. Let $w(t)$ be a function of bounded variation on $\left[T_{0}, \infty\right)$ with some $T_{0}>0$ such that the variation $\mathrm{V}_{a}^{b} w$ on $[a, b]$ satisfies the inequality $\mathrm{V}_{a}^{b} w \leqslant c w(a)$ with a certain constant $c>0$ for any subinterval $[a, b] \subset\left[T_{0}, \infty\right)$. Let

$$
U_{T}=U(T, w)=\int_{T_{0}}^{T} w(t) \mathrm{d} t
$$

and $\operatorname{let}_{T \rightarrow \infty} U(T, w)=+\infty$. Moreover, let $I_{A}$ denote the indicator function of the set $A$. Then we have the following generalization of Theorem 1 .

Theorem 3. Suppose that the function $w(t)$ satisfies the above hypotheses. Let $K \in \mathcal{K}$ and $f(s) \in H_{0}(K)$. Then, for every $\varepsilon>0$,

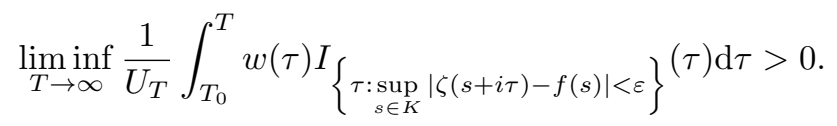

To be precise, in [10], Theorem 3 was proved under a certain additional hypothesis on the function $w(t)$ which is a weighted version of the classical Birkhoff-Khintchine ergodic theorem. In [18], this technical hypothesis was removed. A generalization of Theorem 3 for Matsumoto zeta-functions was given in [12]. In [17], a weighted discrete universality theorem with the sequence $\left\{k^{\alpha} h\right\}, 0<\alpha<1$, for the periodic zeta-function was obtained.

The aim of this paper is a weighted discrete universality theorem for the Riemann zeta-function. Let $w(t)$ be a real non-negative function having a continuous derivative on $\left[\frac{1}{2}, \infty\right)$ such that

$$
\lim _{N \rightarrow \infty} V_{N}=+\infty, \quad V_{N}=\sum_{k=1}^{N} w(k), \quad \int_{1}^{N} u\left|w^{\prime}(u)\right| \mathrm{d} u \ll V_{N}, \quad N \rightarrow \infty .
$$

Denote by $W$ the class of functions $w(t)$ satisfying the above hypotheses. Suppose that $h$ is a fixed positive number.

Theorem 4. Suppose that $w(t) \in W$. Let $K \in \mathcal{K}$ and $f(s) \in H_{0}(K)$. Then, for every $\varepsilon>0$,

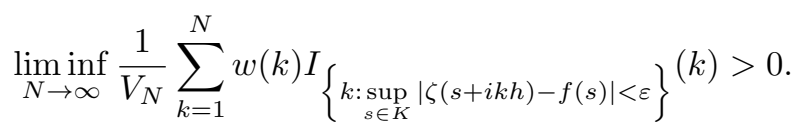

For example, the function $w(t)=\frac{\sin (\log t)+1}{t}$ is not monotonically decreasing and $w(t) \in W$.

Theorem 4 has the following modification. 
Theorem 5. Suppose that $w(t) \in W$. Let $K \in \mathcal{K}$ and $f(s) \in H_{0}(K)$. Then the limit

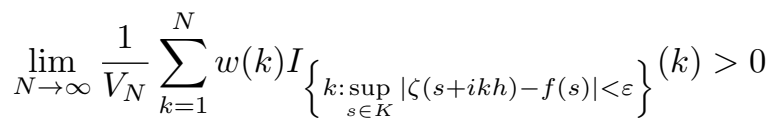

exists for all but at most countably many $\varepsilon>0$.

For proving of the above universality theorems, we will apply the probabilistic approach.

\section{Limit theorems}

We remind that $D=\left\{s \in \mathbb{C}: \frac{1}{2}<\sigma<1\right\}$, and by $H(D)$ denote the space of analytic functions on $D$ endowed with the topology of uniform convergence on compacta. The space $H(D)$ is metrisable. There exists a sequence of compact subsets $\left\{K_{l}: l \in \mathbb{N}\right\} \subset D$ such that $D=\bigcup_{l=1}^{\infty} K_{l}, K_{l} \subset K_{l+1}$ for all $l \in \mathbb{N}$, and if $K \subset D$ is a compact set, then $K \subset K_{l}$ for some $l \in \mathbb{N}$. For $g_{1}, g_{2} \in H(D)$, define

$$
\rho\left(g_{1}, g_{2}\right)=\sum_{l=1}^{\infty} 2^{-l} \frac{\sup _{s \in K}\left|g_{1}(s)-g_{2}(s)\right|}{1+\sup _{s \in K}\left|g_{1}(s)-g_{2}(s)\right|} .
$$

Then $\rho$ is a metric on $H(D)$ which induces its topology of uniform convergence on compacta.

Denote by $\mathcal{B}(X)$ the Borel $\sigma$-field of the space $X$, and, for $A \in \mathcal{B}(H(D))$, define

$$
P_{N}(A)=P_{N, w, h}(A)=\frac{1}{V_{N}} \sum_{k=1}^{N} w(k) I_{\{k: \zeta(s+i k h) \in A\}}(k) .
$$

In this section, we will consider the weak convergence of $P_{N, w, h}$ as $N \rightarrow \infty$. We say that $h>0$ is of type 1 if $\exp \left\{\frac{2 \pi m}{h}\right\}$ is an irrational number for all $m \in \mathbb{Z} \backslash\{0\}$, and $h>0$ is of type 2 if $h$ is not of type 1 . We will examine separately the cases of types 1 and 2 .

As usual, we start with one topological structure. Let $\gamma=\{s \in \mathbb{C}:|s|=1\}$ and $\Omega=\prod_{p} \gamma_{p}$, where $\gamma_{p}=\gamma$ for all primes $p$. By the Tikhonov theorem, the torus $\Omega$ with the product topology and pointwise multiplication is a compact topological Abelian group. Therefore, on $(\Omega, \mathcal{B}(\Omega))$, the probability Haar measure $m_{H}$ can be defined, and this gives the probability space $\left(\Omega, \mathcal{B}(\Omega), m_{H}\right)$. Let $\mathbb{P}$ be the set of all prime numbers, and let $\omega(p)$ denote the projection of $\omega \in \Omega$ to the circle $\gamma_{p}, p \in \mathbb{P}$. For $A \in \mathcal{B}(\Omega)$, define

$$
Q_{N}(A)=\frac{1}{V_{N}} \sum_{k=1}^{N} w(k) I_{\left\{k:\left(p^{-i k h}: p \in \mathbb{P}\right) \in A\right\}}(k) .
$$

Lemma 1. Suppose that $w(t) \in W$ and $h$ is of type 1. Then $Q_{N}$ converges weakly to the Haar measure $m_{H}$ as $N \rightarrow \infty$. 
Proof. We apply the Fourier transform method. Let $g_{N}(\underline{k}), \underline{k}=\left(k_{p}: k_{p} \in\right.$ $\mathbb{Z}, p \in \mathbb{P})$, be the Fourier transform of $Q_{N}$. Then we have that

$$
g_{N}(\underline{k})=\int_{\Omega} \prod_{p}^{*} \omega^{k_{p}}(p) \mathrm{d} Q_{N}
$$

where the sign "** means that only a finite number of integers $k_{p}$ are distinct from zero. Thus, by the definition of $Q_{N}$,

$$
g_{N}(\underline{k})=\frac{1}{V_{N}} \sum_{k=1}^{N} w(k) \prod_{p}^{*} p^{-i k k_{p} h}=\frac{1}{V_{N}} \sum_{k=1}^{N} w(k) \exp \left\{-i k h \sum_{p}^{*} k_{p} \log p\right\} .
$$

Obviously,

$$
g_{N}(\underline{0})=1 \text {. }
$$

If $\underline{k} \neq \underline{0}$, then

$$
\sum_{p}^{*} k_{p} \log p \neq 0
$$

since the logarithms of prime numbers are linearly independent over the field of rational numbers. Thus,

$$
\exp \left\{-i h \sum_{p}^{*} k_{p} \log p\right\} \neq 1
$$

Indeed, if inequality $(2.3)$ is not true, then

$$
\sum_{p}^{*} k_{p} \log p=\frac{2 \pi r}{h}, \quad \prod_{p}^{*} p^{-k_{p}}=\exp \left\{\frac{2 \pi r}{h}\right\}
$$

with some $r \in \mathbb{Z} \backslash\{0\}$. However, the left-hand side of this equality is a rational number, and we arrive to the contradiction that $h$ is of type 1 . Thus, (2.3) is true, and we find that, for $u \geqslant 1$,

$$
\begin{aligned}
\sum_{k \leqslant u} \exp \left\{-i k h \sum_{p}^{*} k_{p} \log p\right\} & \\
= & \frac{\exp \left\{-i h \sum_{p}^{*} k_{p} \log p\right\}-\exp \left\{i([u]+1) h \sum_{p}^{*} k_{p} \log p\right\}}{1-\exp \left\{-i h \sum_{p}^{*} k_{p} \log p\right\}} \stackrel{\text { def }}{=} \Sigma(u) .
\end{aligned}
$$

Hence, in view of $(2.1)$, for $\underline{k} \neq \underline{0}$,

$$
g_{N}(\underline{k})=\frac{w(N) \Sigma(N)}{V_{N}}-\frac{1}{V_{N}} \int_{1}^{N} \Sigma(u) w^{\prime}(u) \mathrm{d} u .
$$

Since the function $\Sigma(u)$ is bounded by a constant not depending of $u$, we find that, for $\underline{k} \neq \underline{0}$,

$$
\lim _{N \rightarrow \infty} g_{N}(\underline{k})=0 .
$$


This together with (2.2) proves the lemma.

Lemma 1 implies a weighted discrete universality theorem for absolutely convergent Dirichlet series. Let $\theta>\frac{1}{2}$ be a fixed number, and

$$
\begin{aligned}
& v_{n}(m)=\exp \left\{-\left(\frac{m}{n}\right)^{\theta}\right\}, \quad m, n \in \mathbb{N}, \\
& \zeta_{n}(s)=\sum_{m=1}^{\infty} \frac{v_{n}(m)}{m^{s}}, \quad \zeta_{n}(s, \omega)=\sum_{m=1}^{\infty} \frac{\omega(m) v_{n}(m)}{m^{s}},
\end{aligned}
$$

where

$$
\omega(m)=\prod_{\substack{p^{\alpha} \mid m \\ p^{\alpha+1} \nmid m}} \omega^{\alpha}(p), \quad m \in \mathbb{N} .
$$

Then the series for $\zeta_{n}(s)$ and $\zeta_{n}(s, \omega)$ are absolutely convergent for $\sigma>\frac{1}{2}$ [11]. From this, it follows that the function $u_{n}: \Omega \rightarrow H(D), u_{n}(\omega)=\zeta_{n}(s, \omega)$, is continuous. Let $R_{n}=m_{H} u_{n}^{-1}$, where

$$
R_{n}(A)=m_{H} u_{n}^{-1}(A)=m_{H}\left(u_{n}^{-1} A\right), \quad A \in \mathcal{B}(H(D)) .
$$

Moreover, let

$$
P_{N, n}(A)=\frac{1}{V_{N}} \sum_{k=1}^{N} w(k) I_{\left\{k: \zeta_{n}(s+i k h) \in A\right\}}(k), \quad A \in \mathcal{B}(H(D)) .
$$

It is not difficult to see that $P_{N, n}=Q_{N} u_{n}^{-1}$. This, the continuity of $u_{n}$ and Lemma 1 lead to

Lemma 2. Suppose that $w(t) \in W$ and $h$ is of type 1. Then $P_{N, n}$ converges weakly to $R_{n}$ as $N \rightarrow \infty$.

The weak convergence of $P_{N, n}$ is a starting point for proving the weak convergence for $P_{N}$ as $N \rightarrow \infty$. The investigation of $P_{N}$ also requires an approximation of $\zeta(s)$ by $\zeta_{n}(s)$. Let $l_{n}(s)=\frac{s}{\theta} \Gamma\left(\frac{s}{\theta}\right) n^{s}$, where $\Gamma(s)$ is the Euler gamma-function. Then [11], for $\sigma>\frac{1}{2}$, the integral representation

$$
\zeta_{n}(s)=\frac{1}{2 \pi i} \int_{\theta-i \infty}^{\theta+i \infty} \zeta(s+z) l_{n}(z) \frac{\mathrm{d} z}{z}
$$

is true. Using the well-known estimates

$$
\int_{1 / 2}^{T}|\zeta(\sigma+i t)|^{2} \mathrm{~d} t \ll T, \quad \int_{1 / 2}^{T}\left|\zeta^{\prime}(\sigma+i t)\right|^{2} \mathrm{~d} t \ll T,
$$

we find that, for $\frac{1}{2}<\sigma<1$ and $\tau \in \mathbb{R}$,

$$
\int_{1 / 2}^{T}|\zeta(\sigma+i t+i \tau)|^{2} \mathrm{~d} t \ll T(1+|\tau|)
$$


and

$$
\int_{1 / 2}^{T}\left|\zeta^{\prime}(\sigma+i t+i \tau)\right|^{2} \mathrm{~d} t \ll T(1+|\tau|) .
$$

These estimates together with Gallagher lemma, see, for example, [20, Lemma 1.4], give, for $\frac{1}{2}<\sigma<1$ and $\tau \in \mathbb{R}$, the bound

$$
\begin{aligned}
& \sum_{k=1}^{N}|\zeta(\sigma+i k h+i \tau)|^{2} \ll \int_{1 / 2}^{(N+1 / 2) h}|\zeta(\sigma+i t+i \tau)|^{2} \mathrm{~d} t \\
& +\left(\int_{1 / 2}^{(N+1 / 2) h} \mid \zeta\left(\sigma+i t+\left.i \tau\right|^{2} \mathrm{~d} t \int_{1 / 2}^{(N+1 / 2) h}\left|\zeta^{\prime}(\sigma+i t+i \tau)\right|^{2} \mathrm{~d} t\right)^{1 / 2} \ll N(1+|\tau|) .\right.
\end{aligned}
$$

Hence, for the same $\sigma$ and $\tau$,

$$
\begin{aligned}
& \sum_{k=1}^{N} w(k)|\zeta(\sigma+i k h+i \tau)|^{2} \ll w(N) \sum_{k=1}^{N}|\zeta(\sigma+i k h+i \tau)|^{2}+(1+|\tau|) \\
& \times \int_{1}^{N} u\left|w^{\prime}(u)\right| \mathrm{d} u \ll N w(N)(1+|\tau|)+V_{N}(1+|\tau|) \ll V_{N}(1+|\tau|),
\end{aligned}
$$

because

$$
N w(N)=\sum_{k=1}^{N} w(k)+\int_{1}^{N}\left(\sum_{k \leqslant u} 1\right) w^{\prime}(u) \mathrm{d} u \ll V_{N} .
$$

Let $K \subset D$ be a compact set. Then (2.4), (2.5), the residue theorem and Cauchy integral formula imply the equality

$$
\lim _{n \rightarrow \infty} \limsup _{N \rightarrow \infty} \frac{1}{V_{N}} \sum_{k=1}^{N} w(k) \sup _{s \in K}\left|\zeta(s+i k h)-\zeta_{n}(s+i k h)\right|=0 .
$$

Now, (2.6) together with the definition of the metric $\rho$ yields the following lemma.

Lemma 3. Suppose that $w(t) \in W$. Then the equality

$$
\lim _{n \rightarrow \infty} \limsup _{N \rightarrow \infty} \frac{1}{V_{N}} \sum_{k=1}^{N} w(k) \rho\left(\zeta(s+i k h), \zeta_{n}(s+i k h)\right)=0
$$

is true for every fixed $h>0$.

Now, we are in position to prove a weighted discrete limit theorem for the function $\zeta(s)$. On the probability space $\left(\Omega, \mathcal{B}(\Omega), m_{H}\right)$, define the $H(D)$-valued random element $\zeta(s, \omega)$ by the Euler product

$$
\zeta(s, \omega)=\prod_{p}\left(1-\frac{\omega(p)}{p^{s}}\right)^{-1} .
$$


The latter product, for almost all $\omega \in \Omega$, is uniformly convergent on compact subsets of the strip $D[11]$. Denote by $P_{\zeta}$ the distribution of the random element $\zeta(s, \omega)$, i.e.,

$$
P_{\zeta}(A)=m_{H}(\omega \in \Omega: \zeta(s, \omega) \in A), \quad A \in \mathcal{B}(H(D)) .
$$

Theorem 6. Suppose that $w(t) \in W$ and $h>0$ is of the type 1. Then $P_{N}$ converges weakly to $P_{\zeta}$ as $N \rightarrow \infty$. Moreover, the support of $P_{\zeta}$ is the set $S=\{g \in H(D): g(s) \neq 0$ or $g(s) \equiv 0\}$.

Proof. We will prove that $R_{n}$, as $n \rightarrow \infty$, converges weakly to a certain probability measure $P$, and that $P_{N}$, as $N \rightarrow \infty$, also converges weakly to $P$.

Let $\theta_{N}$ be a random variable defined on a certain probability space with probability measure $\mu$ and having the distribution

$$
\mu\left(\theta_{N}=k h\right)=\frac{w(k)}{V_{N}}, \quad k=1, \ldots, N .
$$

Moreover, let $Y_{N, n}=Y_{N, n}(s)$ be an $H(D)$-valued random element defined by

$$
Y_{N, n}(s)=\zeta_{n}\left(s+i \theta_{N}\right),
$$

and let $Y_{n}=Y_{n}(s)$ be an $H(D)$-valued random element with the distribution $R_{n}$. Then, by Lemma 2 ,

$$
Y_{N, n} \underset{N \rightarrow \infty}{\stackrel{\mathcal{D}}{\longrightarrow}} Y_{n}
$$

Using the absolute convergence of the series for $\zeta_{n}(s)$, it can be proved by a method of [11] that the family of probability measures $\left\{R_{n}: n \in \mathbb{N}\right\}$ is tight, i.e., for every $\varepsilon>0$, there exists a compact set $K=K(\varepsilon) \subset H(D)$ such that

$$
R_{n}(K)>1-\varepsilon
$$

for all $n \in \mathbb{N}$. Hence, by the Prokhorov theorem [2], this family is relatively compact. Therefore, each sequence of $\left\{R_{n}\right\}$ contains a subsequence $\left\{R_{n_{r}}\right\}$ weakly convergent, as $r \rightarrow \infty$, to a certain probability measure $P$ on $(H(D), \mathcal{B}(H(D)))$. In other words,

$$
Y_{n_{r}} \underset{r \rightarrow \infty}{\stackrel{\mathcal{D}}{\longrightarrow}} P .
$$

Define one more $H(D)$-valued random element

$$
X_{N}=X_{N}(s)=\zeta\left(s+i \theta_{N}\right) .
$$

Then the application of Lemma 3 gives, for $\varepsilon>0$,

$$
\begin{aligned}
\lim _{n \rightarrow \infty} & \limsup _{N \rightarrow \infty} \mu\left(\rho\left(X_{N}(s), Y_{N, n}(s)\right) \geqslant \varepsilon\right) \\
& =\lim _{n \rightarrow \infty} \limsup _{N \rightarrow \infty} \frac{1}{V_{N}} \sum_{k=1}^{N} w(k) I_{\left\{k: \rho\left(\zeta(s+i k h), \zeta_{n}(s+i k h)\right) \geqslant \varepsilon\right\}}(k) \\
& \leqslant \lim _{n \rightarrow \infty} \limsup _{N \rightarrow \infty} \frac{1}{\varepsilon V_{N}} \sum_{k=1}^{N} w(k) \rho\left(\zeta(s+i k h), \zeta_{n}(s+i k h)\right)=0 .
\end{aligned}
$$


This equality, (2.7) and (2.8) show that all hypotheses of Theorem 4.2 of [2] are satisfied, therefore,

$$
X_{N} \underset{N \rightarrow \infty}{\stackrel{\mathcal{D}}{\longrightarrow}} P
$$

or $P_{N}$ converges weakly to $P$ as $N \rightarrow \infty$. Moreover, in virtue of $(2.9)$, the measure $P$ is independent of the sequence $Y_{n_{r}}$. Since the family $\left\{R_{n}\right\}$ is relatively compact, from this, we obtain that $R_{n}$ converges weakly to $P$ as $n \rightarrow \infty$. Thus, $P_{N}$, as $N \rightarrow \infty$, converges weakly to the limit measure $P$ of $R_{n}$ as $n \rightarrow \infty$. However, by the proof of a limit theorem for

$$
\frac{1}{T} \operatorname{meas}\{\tau \in[0, T]: \zeta(s+i \tau) \in A\}, \quad A \in \mathcal{B}(H(D)),
$$

it is known [11] that $R_{n}$, as $n \rightarrow \infty$, converges weakly to $P_{\zeta}$, and the support of $P_{\zeta}$ is the set $S$. Therefore, the same statement is also true for $P_{N}$, and the theorem is proved.

The case of $h$ of type 2 is a more complicated. We must construct a new probability space different from $\left(\Omega, \mathcal{B}(\Omega), m_{H}\right)$. We will index by $h$ the notation related to $h$ of type 2 .

Now suppose that $h>0$ is of type 2 . Then there exists the smallest $m_{0} \in \mathbb{N}$ such the number $\exp \left\{\frac{2 \pi m_{0}}{h}\right\}$ is rational. We put

$$
\exp \left\{\frac{2 \pi m_{0}}{h}\right\}=\frac{a}{b}, \quad a, b \in \mathbb{N},(a, b)=1 .
$$

Define the set

$$
\mathbb{P}_{0}=\left\{p \in \mathbb{P}: \frac{a}{b}=\prod_{p \in \mathbb{P}} p^{\alpha_{p}} \text { with } \alpha_{p} \neq 0\right\}
$$

Denote by $\Omega_{h}$ the closed subgroup of $\Omega$ generated by the element $\left\{p^{-i h}: p \in \mathbb{P}\right\}$. By Lemma 1 of [14], if $h$ is of type 2, then

$$
\Omega_{h}=\{\omega \in \Omega: \omega(a)=\omega(b)\} .
$$

On $\left(\Omega_{h}, \mathcal{B}\left(\Omega_{h}\right)\right)$, the probability Haar measure $m_{H}^{h}$ exists, and we obtain the probability space $\left(\Omega_{h}, \mathcal{B}\left(\Omega_{h}\right), m_{H}^{h}\right)$. By (3.1) of [14], we have that the characters $\chi$ of the group $\Omega_{h}$ are of the form

$$
\chi(\omega)=\prod_{p \in \mathbb{P} \backslash \mathbb{P}_{0}}^{*} \omega^{k_{p}}(p) \prod_{p \in \mathbb{P}_{0}} \omega^{k_{p}+l \alpha_{p}}(p), \quad l \in \mathbb{Z} .
$$

Now, we are ready to prove an analogue of Lemma 1 for $h$ of type 2 . For $A \in \mathcal{B}\left(\Omega_{h}\right)$, define

$$
Q_{N, h}(A)=\frac{1}{V_{N}} \sum_{k=1}^{N} w(k) I_{\left\{k:\left(p^{-i k h}: p \in \mathbb{P}\right) \in A\right\}}(k) .
$$

Lemma 4. Suppose that $h$ is of type 2. Then $Q_{N, h}$ converges weakly the Haar measure $m_{H}^{h}$ as $N \rightarrow \infty$. 
Proof. In view of (2.10), we have that the Fourier transform $g_{N, h}(\underline{k}), \underline{k}=$ $\left(k_{p}: k_{p} \in \mathbb{Z}, p \in \mathbb{P}\right)$, of $Q_{N, h}$ is of the form

$$
\begin{aligned}
g_{N, h}(\underline{k}) & =\int_{\Omega_{h}} \chi(\omega) \mathrm{d} Q_{N, h} \\
& =\frac{1}{V_{N}} \sum_{k=1}^{N} w(k) \prod_{p \in \mathbb{P} \backslash \mathbb{P}_{0}}^{*} p^{-i k k_{p} h} \prod_{p \in \mathbb{P}_{0}} p^{-i k h\left(k_{p}+l \alpha_{p}\right)}, \quad l \in \mathbb{Z} .
\end{aligned}
$$

If $k_{p}=0$ for all $p \in \mathbb{P} \backslash \mathbb{P}_{0}$ and $k_{p}=r \alpha_{p}$ for all $p \in \mathbb{P}_{0}$ with some $r \in \mathbb{Z}$ (case 1 ), then

$$
g_{N, h}(\underline{k})=1,
$$

because $\prod_{p \in \mathbb{P}_{0}} \omega^{d \alpha_{p}}(p)=1$ with $d \in \mathbb{Z}$.

Now, suppose that $k_{p} \neq 0$ for some $p \in \mathbb{P} \backslash \mathbb{P}_{0}$, or there does not exist $r \in \mathbb{Z}$ such that $k_{p}=r \alpha_{p}$ for all $p \in \mathbb{P}_{0}$ (case 2). In [14], it was obtained that

$$
\exp \left\{-i h A_{p}\left(k_{p}, l \alpha_{p}\right)\right\} \neq 1 \text {, }
$$

where

$$
A_{p}\left(k_{p}, l \alpha_{p}\right)=\sum_{p \in \mathbb{P} \backslash \mathbb{P}_{0}}^{*} k_{p} \log p+\sum_{p \in \mathbb{P}_{0}}\left(k_{p}+l \alpha_{p}\right) \log p, \quad l \in \mathbb{Z} .
$$

Hence, we find that, for $u \geqslant 1$,

$$
\begin{aligned}
& \sum_{k \leqslant u} \exp \left\{-i k h A_{p}\left(k_{p}, l \alpha_{p}\right)\right\} \\
& \quad=\frac{\exp \left\{-i h A_{p}\left(k_{p}, l \alpha_{p}\right)\right\}-\exp \left\{-i h([u]+1) A_{p}\left(k_{p}, l \alpha_{p}\right)\right\}}{1-\exp \left\{-i h A_{p}\left(k_{p}, l \alpha_{p}\right)\right\}} \stackrel{\text { def }}{=} \Sigma_{h}(u) .
\end{aligned}
$$

Therefore, in view of (2.11),

$$
g_{N, h}(\underline{k})=\frac{w(N) \Sigma_{h}(N)}{V_{N}}-\frac{1}{V_{N}} \int_{1}^{N} \Sigma_{h}(u) w^{\prime}(u) \mathrm{d} u .
$$

Using the properties of the function $w$, hence we find that

$$
g_{N, h}(\underline{k})=0 .
$$

This together with (2.12) shows that

$$
\lim _{N \rightarrow \infty} g_{N, h}(\underline{k})= \begin{cases}1, & \text { in the case } 1, \\ 0, & \text { in the case } 2 .\end{cases}
$$

Since the right-hand side of the equality is the Fourier transform of the Haar measure $m_{H}^{h}$, the lemma follows by a continuity theorem for probability measures on compact groups.

Now, together with $P_{N, n, h}$, consider

$$
\hat{P}_{N, n, h}(A)=\frac{1}{V_{N}} \sum_{k=1}^{N} w(k) I_{\left\{k: \zeta_{n, h}(s+i k h, \omega) \in A\right\}}(k), \quad A \in \mathcal{B}(H(D)),
$$

with $\omega \in \Omega_{h}$. 
Lemma 5. Suppose that $w(t) \in W$ and $h$ is of type 2. Then $P_{N, n, h}$ and $\hat{P}_{N, n, h}$ both converge weakly to the measure $m_{H}^{h} u_{n, h}^{-1}$ as $N \rightarrow \infty$, where $u_{n, h}: \Omega_{h} \rightarrow$ $H(D)$ is given by $u_{n, h}(\omega)=\zeta_{n, h}(s, \omega), \omega \in \Omega_{h}$.

Proof. By proving Lemma 2, in view of Lemma 4, we have that $P_{N, n, h}$ converges weakly to $m_{H}^{h} u_{n, h}^{-1}$ as $N \rightarrow \infty$. Similarly, we obtain that if $\hat{u}_{n, h}(\hat{\omega})$ : $\Omega_{h} \rightarrow H(D)$ is given by

$$
\hat{u}_{n, h}(\hat{\omega})=\zeta_{n}(s, \omega \hat{\omega}), \quad \hat{\omega} \in \Omega_{h},
$$

then $\hat{P}_{N, n, h}$ converges weakly to $m_{H}^{h} \hat{u}_{n, h}^{-1}$. However, $\hat{u}_{n, h}=u_{n, h}(u)$, where $u: \Omega_{h} \rightarrow \Omega_{h}$ is given by $u(\hat{\omega})=\omega \hat{\omega}$. This and the invariance of the Haar measure $m_{H}^{h}$ show that $m_{H}^{h} \hat{u}_{n, h}^{-1}=m_{H}^{h} u_{n, h}^{-1}$.

For further considerations, we need some elements of the ergodic theory. Let $a_{h}=\left(p^{-i h}: p \in \mathbb{P}\right)$. Then $a_{h}$ is an element of $\Omega_{h}$. Define the transformation $\varphi_{h}(\omega)$ of $\Omega_{h}$ by

$$
\varphi_{h}(\omega)=a_{h} \omega, \quad \omega \in \Omega_{h} .
$$

Then we have that $\varphi_{h}$ is a measurable measure preserving transformation on the probability space $\left(\Omega_{h}, \mathcal{B}\left(\Omega_{h}\right), m_{H}^{h}\right)$. We recall that a set $A \in \mathcal{B}\left(\Omega_{h}\right)$ is called invariant with respect to $\varphi_{h}$ if the sets $A$ and $\varphi_{h}(A)$ can differ from each other at most by a set of $m_{H}^{h}$-measure zero. The transformation $\varphi_{h}$ is called ergodic if the $\sigma$-field of invariant sets of $\Omega_{h}$ consists only of the sets having $m_{H}^{h}$-measure 1 or 0 .

Lemma 6. Suppose that $h$ is of type 2. Then the transformation $\varphi_{h}$ is ergodic.

Proof of the lemma is given in [14, Lemma 3].

Let, for $\omega \in \Omega_{h}$,

$$
\zeta_{h}(s, \omega)=\prod_{p}\left(1-\frac{\omega(p)}{p^{s}}\right)^{-1} .
$$

The first application of Lemma 6 is devoted to the discrete mean square of $\zeta_{h}(s, \omega)$.

Lemma 7. Suppose that $w(t) \in W, h>0$ is of type 2, $\sigma, \frac{1}{2}<\sigma<1$, is fixed and $t \in \mathbb{R}$. Then, for almost all $\omega \in \Omega_{h}$,

$$
\sum_{k=1}^{N} w(k)\left|\zeta_{h}(\sigma+i t+i k h, \omega)\right|^{2} \ll V_{N}(1+|t|) .
$$

Proof. We have that $\zeta_{h}(s, \omega)$ coincides with the restriction of the random element $\zeta(s, \omega)$ to the space $\left(\Omega_{h}, \mathcal{B}\left(\Omega_{h}\right), m_{H}^{h}\right)$. First we consider the expectation $\mathbb{E}\left|\zeta_{h}(\sigma+i t, \omega)\right|^{2}$. We write $\zeta_{h}(s, \omega)$ in the form

$$
\zeta_{h}(\sigma+i t, \omega)=\prod_{p \in \mathbb{P}_{0}}\left(1-\frac{\omega(p)}{p^{\sigma+i t}}\right)^{-1} \prod_{p \in \mathbb{P} \backslash \mathbb{P}_{0}}\left(1-\frac{\omega(p)}{p^{\sigma+i t}}\right)^{-1} \stackrel{\text { def }}{=} X_{1} X_{2} .
$$


The random elements $X_{1}$ and $X_{2}$ are independent, moreover, for almost all $\omega \in \Omega_{h}$,

$$
X_{2}=\sum_{m}^{\prime} \frac{\omega(m)}{m^{\sigma+i t}}
$$

where the sign ' means that the summing runs over $m=1$ and $m \in \mathbb{N}$ with the canonical representation consisting only of primes $p \in \mathbb{P} \backslash \mathbb{P}_{0}$. In the series for $X_{2}$, the random variables are orthogonal, therefore,

$$
\mathbb{E}\left|X_{2}\right|^{2}=\sum_{m}^{\prime} \frac{1}{m^{2 \sigma}}<\infty .
$$

Clearly, $\mathbb{E}\left|X_{1}\right|^{2}$ is bounded by a constant. Therefore, there exists a finite constant $c>0$ such that, for $\frac{1}{2}<\sigma<1$ and $t \in \mathbb{R}$,

$$
\mathbb{E}\left|\zeta_{h}(\sigma+i t, \omega)\right|^{2}=\mathbb{E}\left|X_{1}\right|^{2} \mathbb{E}\left|X_{2}\right|^{2} \leqslant c .
$$

Then (2.13), Lemma 6, the Birkhoff-Khintchine ergodic theorem, see, for example, [23], and the definition of the transformation $\varphi_{h}$ show that, for $\frac{1}{2}<\sigma<1$ and $\left|t_{0}\right| \leqslant h$,

$$
\begin{aligned}
\sum_{k=1}^{N}\left|\zeta_{h}\left(\sigma+i t_{0}+i k h, \omega\right)\right|^{2} & =\sum_{k=1}^{N}\left|\zeta_{h}\left(\sigma+i t_{0}, \varphi_{h}^{k}(\omega)\right)\right|^{2} \\
& =N \mathbb{E}\left|\zeta_{h}\left(\sigma+i t_{0}, \omega\right)\right|^{2}(1+o(1)) \ll N
\end{aligned}
$$

for almost all $\omega \in \Omega_{h}$ as $N \rightarrow \infty$. Hence, denoting by $[u]$ the integer part of $u \in \mathbb{R}$, for $\frac{1}{2}<\sigma<1$ and $t \in \mathbb{R}$, we find that

$$
\sum_{k=1}^{N}\left|\zeta_{h}(\sigma+i t+i k h, \omega)\right|^{2}=\sum_{k=1+[t / h]}^{N+[t / h]}\left|\zeta_{h}\left(\sigma+i t_{0}+i k h, \omega\right)\right|^{2} \ll N(1+|t|)
$$

for almost all $\omega \in \Omega_{h}$. From this, summing by parts, we obtain the estimate of the lemma.

Similarly to the proof of Lemma 3, we arrive, by using Lemma 7, to

Lemma 8. Suppose that $w(t) \in W$ and $h>0$ is of type 2. Then, for almost all $\omega \in \Omega_{h}$,

$$
\lim _{n \rightarrow \infty} \limsup _{N \rightarrow \infty} \frac{1}{V_{N}} \sum_{k=1}^{N} w(k) \rho\left(\zeta_{h}(s+i k h, \omega), \zeta_{n, h}(s+i k h, \omega)\right)=0 .
$$

For $\omega \in \Omega_{h}$, additionally to the measure $P_{N, h}$, define

$$
\hat{P}_{N, h}(A)=\frac{1}{V_{N}} \sum_{k=1}^{N} w(k) I_{\left\{k: \zeta_{h}(s+i k h, \omega) \in A\right\}}(k), \quad A \in \mathcal{B}(H(D)) .
$$

Then, using Lemmas 3,5 and 8, and repeating the first part of the proof of Theorem 6 , we obtain 
Lemma 9. Suppose that $w(t) \in W$ and $h>0$ is of type 2. Then, on $(H(D)$, $\mathcal{B}(H(D)))$, there exists a probability measure $P_{h}$ such that $P_{N, h}$ and $\hat{P}_{N, h}$ both converges weakly to $P_{h}$ as $N \rightarrow \infty$.

Denote by $P_{\zeta, h}$ the distribution of the random element $\zeta_{h}(s, \omega), \omega \in \Omega_{h}$. Then we have the following analogue of Theorem 6 .

Theorem 7. Suppose that $w(t) \in W$ and $h>0$ is of type 2. Then $P_{N, h}$ converges weakly $P_{\zeta, h}$ as $N \rightarrow \infty$. Moreover, the support of the measure $P_{\zeta, h}$ is the set $S$.

Proof. In virtue of Lemma 9, it suffices to identify the measure $P$ in that lemma, and to find the support of the limit measure. For the first problem, we will apply Lemma 6 , and the Birkhoff-Khintchine theorem. Let $A$ be a continuity set of $P$. On the probability space $\left(\Omega_{h}, \mathcal{B}\left(\Omega_{h}\right), m_{H}^{h}\right)$, define the random variable $\xi$ by the formula

$$
\xi(\omega)= \begin{cases}1, & \text { if } \zeta_{h}(s, \omega) \in A \\ 0, & \text { otherwise }\end{cases}
$$

Then we have that

$$
\mathbb{E} \xi=\int_{\Omega_{h}} \xi(\omega) \mathrm{d} m_{H}^{h}=P_{\zeta, h}(A) .
$$

Moreover, by Lemma 9,

$$
\lim _{N \rightarrow \infty} \hat{P}_{N}(A)=P_{h}(A) .
$$

In view of Lemma 6 and the Birkhoff-Khintchine theorem, for almost all $\omega \in$ $\Omega_{h}$,

$$
\lim _{N \rightarrow \infty} \frac{1}{N} \sum_{k=1}^{N} \xi\left(\varphi_{h}^{k}(\omega)\right)=\mathbb{E} \xi
$$

Since $w \in W$, from this it follows that, for almost all $\omega \in \Omega_{h}$,

$$
\lim _{N \rightarrow \infty} \frac{1}{V_{N}} \sum_{k=1}^{N} w(k) \xi\left(\varphi_{h}^{k}(\omega)\right)=\mathbb{E} \xi
$$

However, by the definition of $\varphi_{h}$,

$$
\frac{1}{V_{N}} \sum_{k=1}^{N} w(k) \xi\left(\varphi_{h}^{k}(\omega)\right)=\frac{1}{V_{N}} \sum_{k=1}^{N} w(k) I_{\left\{k: \zeta_{h}(s+i k h, \omega) \in A\right\}}(k)=\hat{P}_{N, h}(A) .
$$

Therefore, by (2.14) and (2.16),

$$
\lim _{N \rightarrow \infty} \hat{P}_{N, h}(A)=P_{\zeta, h}(A) .
$$

This and (2.15) show that $P_{h}=P_{\zeta, h}$.

For finding the support of $P_{\zeta, h}$, we use the representation (2.13). For $p \in$ $\mathbb{P} \backslash \mathbb{P}_{0}$, the random variables $\omega(p)$ are independent. Thus, by the proof of Lemma 6.5.5 from [11], we find that the support of the random element $X_{2}$ is the set $S$. Since the random elements $X_{1}$ and $X_{2}$ are independent and $X_{1}$ is not degenerate at zero, we obtain that the support of $X_{1} X_{2}$ is the set $S$, i.e., the support of the measure $P_{\zeta, h}$ is the set $S$. The theorem is proved. 


\section{Proof of universality theorems}

Theorems 4 and 5 follow from the limit theorems (Theorems 6 and 7), for $\zeta(s)$ as well as from the Mergelyan theorem [19] on the approximation of analytic functions by polynomials.

Proof. (Of Theorem 4). By the Mergelyan theorem, there exists a polynomial $p(s)$ such that

$$
\sup _{s \in K}\left|f(s)-e^{p(s)}\right|<\frac{\varepsilon}{2} .
$$

For brevity, denote the limit measure in Theorems 6 and 7 by $\hat{P}_{\zeta}$, i.e.,

$$
\hat{P}_{\zeta}=\left\{\begin{array}{ll}
P_{\zeta}, & \text { if } h \text { is of type } 1, \\
P_{\zeta, h}, & \text { if } h \text { is of type } 2,
\end{array} \quad \hat{P}_{N}= \begin{cases}P_{N}, & \text { if } h \text { is of type } 1, \\
P_{N, h}, & \text { if } h \text { is of type } 2 .\end{cases}\right.
$$

Then we have that $\hat{P}_{N}$ converges weakly to $\hat{P}_{\zeta}$ as $N \rightarrow \infty$. Define the set

$$
G_{\varepsilon}=\left\{g \in H(D): \sup _{s \in K}\left|g(s)-e^{p(s)}\right|<\frac{\varepsilon}{2}\right\} \text {. }
$$

Since $e^{p(s)} \neq 0$, and, in view of Theorems 6 and 7 , the support of the measure $\hat{P}_{\zeta}$ is the set $S$, the set $G_{\varepsilon}$ is an open neighbourhood of an element of the support, therefore,

$$
\hat{P}_{\zeta}\left(G_{\varepsilon}\right)>0 \text {. }
$$

Moreover, by the first parts of Theorems 6 and 7, and the equivalent of weak convergence of probability measures in terms of open sets [2, Theorem 2.1], we have that

$$
\liminf _{N \rightarrow \infty} \hat{P}_{N}\left(G_{\varepsilon}\right) \geqslant \hat{P}_{\zeta}\left(G_{\varepsilon}\right) .
$$

This, (3.2) and the definitions of $\hat{P}_{N}$ and $G_{\varepsilon}$ show that

$$
\liminf _{N \rightarrow \infty} \frac{1}{V_{N}} \sum_{k=1}^{N} w(k) I_{\left\{k: \sup _{s \in K}\left|\zeta(s+i k h)-e^{p(s)}\right|<\frac{\varepsilon}{2}\right\}}(k)>0 .
$$

It remains to replace $e^{p(s)}$ by $f(s)$ in the latter inequality. Suppose that $k$ satisfies the inequality

$$
\sup _{s \in K}\left|\zeta(s+i k h)-e^{p(s)}\right|<\frac{\varepsilon}{2} .
$$

Then, in virtue of (3.1), the same $k$ satisfies the inequality

$$
\sup _{s \in K}|\zeta(s+i k h)-f(s)|<\varepsilon .
$$

Therefore,

$$
\left\{k: \sup _{s \in K}\left|\zeta(s+i k h)-e^{p(s)}\right|<\frac{\varepsilon}{2}\right\} \subset\left\{k: \sup _{s \in K}|\zeta(s+i k h)-f(s)|<\varepsilon\right\} .
$$


This inclusion together with (3.3) proves the theorem.

Proof. (Of Theorem 5). Define the set

$$
\hat{G}_{\varepsilon}=\left\{g \in H(D): \sup _{s \in K}|g(s)-f(s)|<\varepsilon\right\} .
$$

Then $\partial \hat{G}_{\varepsilon}=\left\{g \in H(D): \sup _{s \in K}|g(s)-f(s)|=\varepsilon\right\}$ is the boundary of $\hat{G}_{\varepsilon}$. Hence, $\partial \hat{G}_{\varepsilon_{1}} \cap \partial \hat{G}_{\varepsilon_{2}}=\varnothing$ if $\varepsilon_{1} \neq \varepsilon_{2}, \varepsilon_{1}>0, \varepsilon_{2}>0$. Therefore, the set $\partial \hat{G}_{\varepsilon}$ can have a positive $\hat{P}_{\zeta}$-measure for at most countably many $\varepsilon>0$. This means that the set $\hat{G}_{\varepsilon}$ is a continuity set of the measure $\hat{P}_{\zeta}$ for all but at most countably many $\varepsilon>0$. Using Theorems 6 and 7 , and the equivalent of weak convergence of probability measures in terms of continuity sets [2, Theorem 2.1], we have that

$$
\lim _{N \rightarrow \infty} \hat{P}_{N}\left(\hat{G}_{\varepsilon}\right)=\hat{P}_{\zeta}\left(\hat{G}_{\varepsilon}\right)
$$

for all but at most countably many $\varepsilon>0$. Moreover, (3.1) shows that $G_{\varepsilon} \subset \hat{G}_{\varepsilon}$. Therefore, by $(3.2), \hat{P}_{\zeta}\left(\hat{G}_{\varepsilon}\right)>0$. This, (3.4) and the definition of the set $\hat{G}_{\varepsilon}$ prove the theorem.

\section{Acknowledgements}

The research of the first author is funded by the European Social Fund according to the activity "Improvement of researchers' qualification by implementing world-class R\&D projects" of Measure No. 09.3.3-LMT-K-712-01-0037.

\section{References}

[1] B. Bagchi. The Statistical Behavior and Universality Properties of the Riemann Zeta-Function and Other Allied Dirichlet Series. PhD Thesis, Indian Statistical Institute, Calcutta, 1981.

[2] P. Billingsley. Convergence of Probability Measures. Willey, New York, 1968.

[3] K.M. Bitar, N.N. Khuri and H.C. Ren. Path integrals and Voronin's theorem on the universality of the Riemann zeta-function. Ann. Phys., 211(1):172-196, 1991. https://doi.org/10.1016/0003-4916(91)90196-F.

[4] H. Bohr. Über das verhalten von $\zeta(s)$ in der halbebene $\sigma>1$. Nachr. Akad. Wiss. Göttingen II Math. Phys. Kl., pp. 409-428, 1911.

[5] H. Bohr and R. Courant. Neue anwendungen der theorie der diophantischen approximationen auf die Riemannsche zetafunktion. Reine Angew. Math., 1914(144):249-274, 1914. https://doi.org/10.1515/crll.1914.144.249.

[6] A. Dubickas and A. Laurinčikas. Distribution modulo 1 and the discrete universality of the Riemann zeta-function. Abh. Math. Semin. Univ. Hamb., 86(1):7987, 2016. https://doi.org/10.1007/s12188-016-0123-8.

[7] R. Garunkštis and A. Laurinčikas. Riemann hypothesis and universality of the Riemann zeta-function. Math. Slovaca, 68(4):741-748, 2018. https://doi.org/10.1515/ms-2017-0141. 
[8] R. Garunkštis, A. Laurinčikas and R. Macaitienè. Zeros of the Riemann zeta-function and its universality. Acta Arith., 181:127-142, 2017. https://doi.org/10.4064/aa8583-5-2017.

[9] S.M. Gonek. Analytic Properties of Zeta and L-Functions. PhD Thesis, University of Michigan, 1979.

[10] A. Laurinčikas. On the universality of the Riemann zeta-function. Lith. Math. J., 35(4):399-402, 1995. https://doi.org/10.1007/BF02335599.

[11] A. Laurinčikas. Limit Theorems for the Riemann Zeta-Function. Kluwer, Dordrecht, 1996.

[12] A. Laurinčikas. On the Matsumoto zeta-function. Acta Arith., 84:1-16, 1998. https://doi.org/10.4064/aa-84-1-1-16.

[13] A. Laurinčikas. Discrete universality of the Riemann zeta-function and uniform distribution modulo 1. St. Petersburg Math. J., 30:103-110, 2019. https://doi.org/10.1090/spmj/1532.

[14] A. Laurinčikas, K. Matsumoto and J. Steuding. Discrete universality of $L$-functions of new forms. II. Lith. Math. J., 56(2):207-218, 2016. https://doi.org/10.1007/s10986-016-9314-3.

[15] A. Laurinčikas and J. Petuškinaitè. Universality of Dirichlet $L$-functions and non-trivial zeros of the Riemann zeta-function. Sb. Math., 210, 2019. https://doi.org/10.1070/SM9194.

[16] R. Macaitienè. On discrete universality of the Riemann zeta-function with respect to uniformly distributed shifts. Arch. Math., 108(3):271-281, 2017. https://doi.org/10.1007/s00013-016-0998-8.

[17] R. Macaitienè, M. Stoncelis and D. Šiaučiūnas. A weighted discrete universality theorem for periodic zeta-functions. II. Math. Modell. Analysis, 22(6):750-762, 2017. https://doi.org/10.3846/13926292.2017.1365779.

[18] R. Macaitienè, M. Stoncelis and D. Šiaučiūnas. A weighted universality theorem for periodic zeta-functions. Math. Modell. Analysis, 22(1):95-105, 2017. https://doi.org/10.3846/13926292.2017.1269373.

[19] S.N. Mergelyan. Uniform approximations to functions of complex variable. Usp. Mat. Nauk., 7:31-122, 1952 (in Russian).

[20] H.L. Montgomery. Topics in Multiplicative Number Theory. Lecture Notes Math. Vol. 227, Springer-Verlag, Berlin, 1971. https://doi.org/10.1007/BFb0060851.

[21] Ł. Pańkowski. Joint universality for dependent $L$-functions. Ramanujan J., 45(1):181-195, 2018. https://doi.org/10.1007/s11139-017-9886-5.

[22] A. Reich. Werteverteilung von zetafunktionen. Arch. Math., 34(1):440-451, 1980. https://doi.org/10.1007/BF01224983.

[23] A.N. Shiryaev. Probability. Graduate Texts Math. vol. 95, Springer-Verlag, New York, 1984.

[24] J. Steuding. Value-Distribution of L-Functions. Lecture Notes Math. vol. 1877, Springer, Berlin, Heidelberg, 2007.

[25] S.M. Voronin. On the distribution of nonzero values of the Riemann $\zeta$-function. Trudy Mat. Inst. Steklov, 128:131-150, 1972 (in Russian).

[26] S.M. Voronin. Theorem on the "universality" of the Riemann zeta-function. Izv. Akad. Nauk SSSR, Ser. Matem., 39:475-486, 1975 (in Russian). 